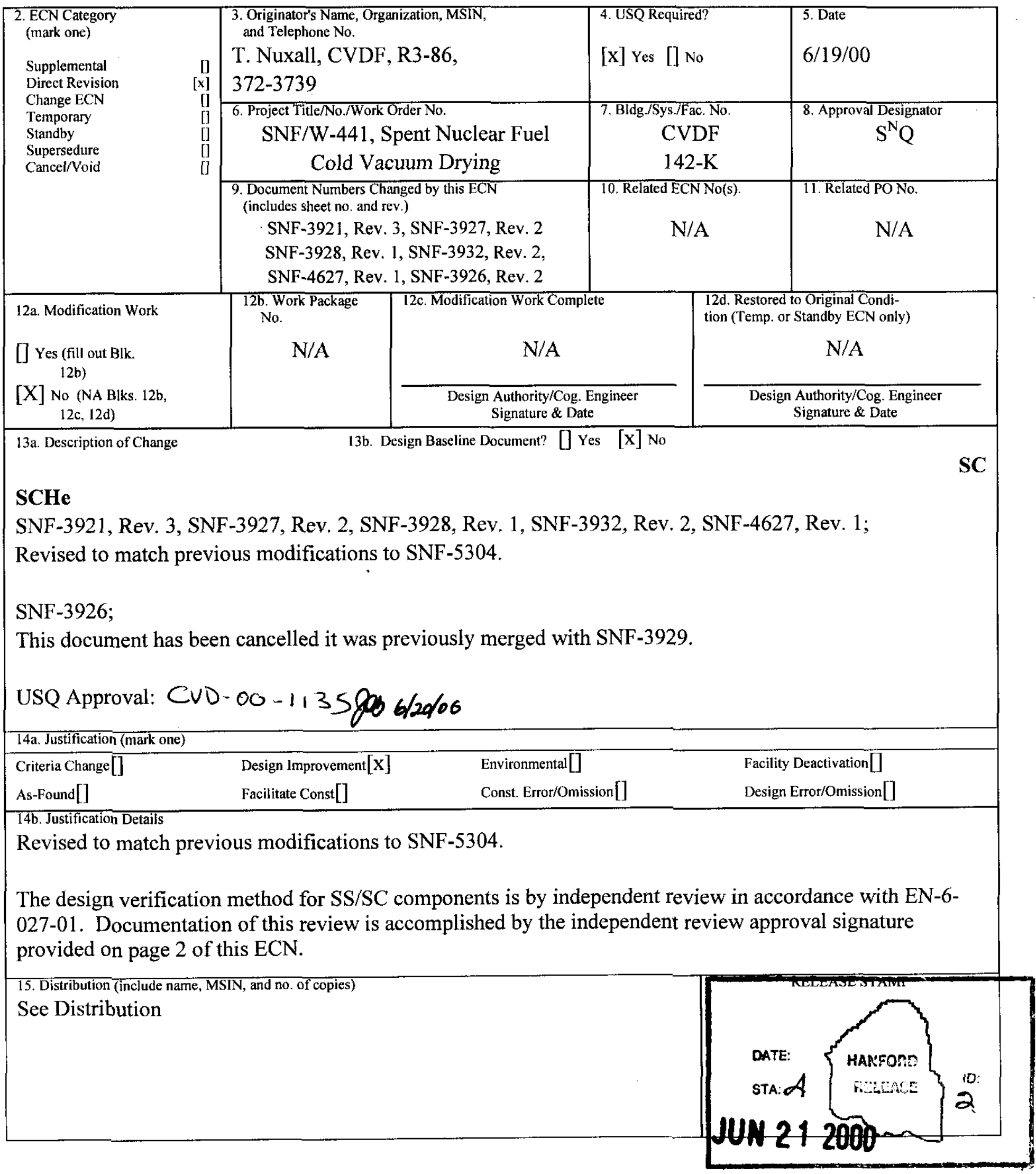




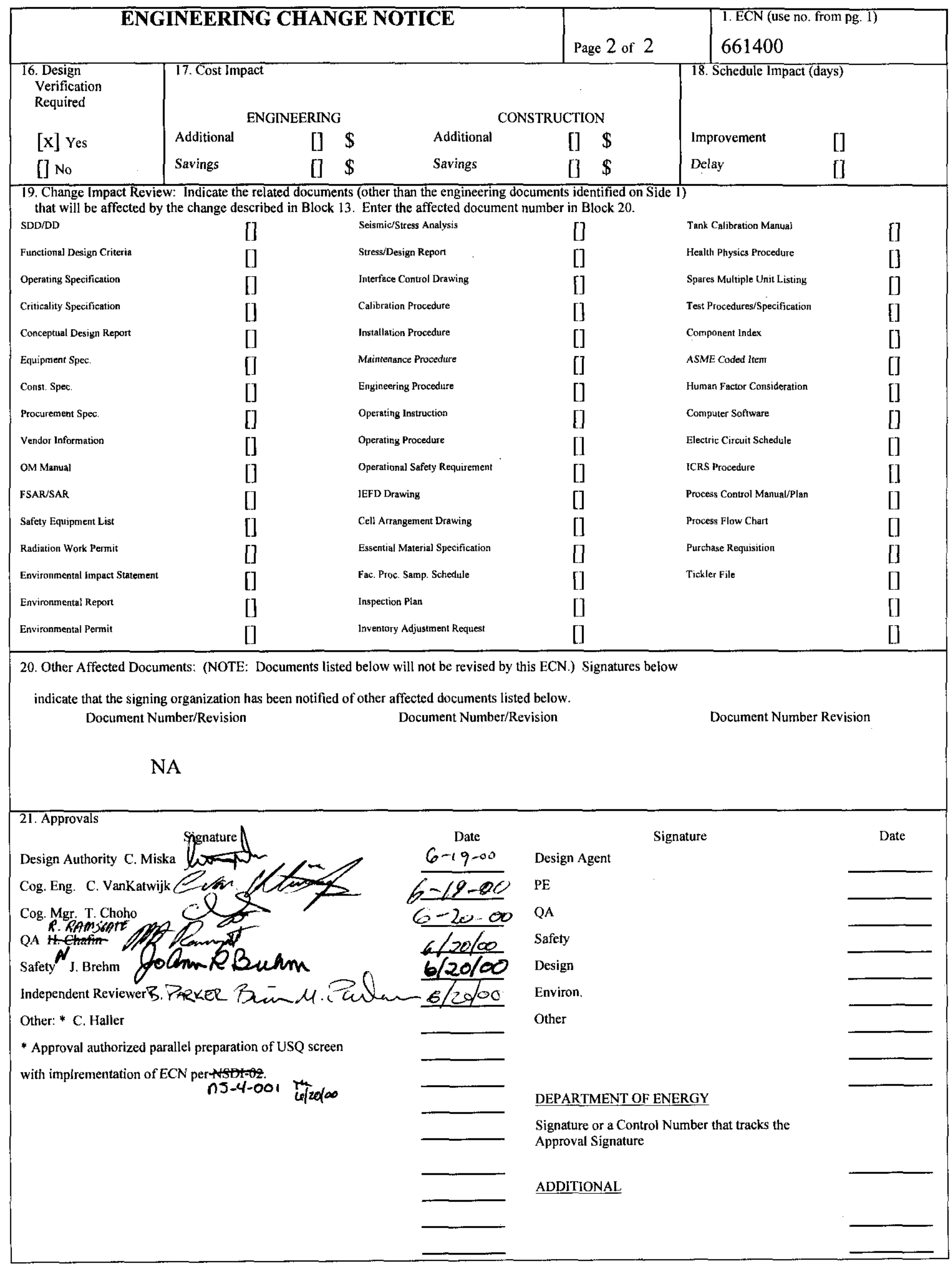




\section{Ashcroft Pressure Switch - Monitor for Flow SCHe Supply Bottle Pressure}

Prepared for the U.S. Department of Energy

Assistant Secretary for Environmental Management

Project Hanford Management Contractor for the

U.S. Department of Energy under Contract DE-AC06-96RL13200

Fluor Hanford

P.O. Box 1000

Richland, Washington 
SNF-3927

Revision 3

ECN 661400

\section{Ashcroft Pressure Switch - Monitor for Flow SCHe Supply Bottle Pressure}

Project No: W-441

Document Type: RPT

Division: SNF

C Van Katwijk

$\mathrm{FH}$

Date Published

June 2000

Prepared for the U.S. Department of Energy

Assistant Secretary for Environmental Management

Project Hanford Management Contractor for the

U.S. Department of Energy under Contract DE-AC06-96RL13200

\section{Fluor Hanford}

P.O. Box 1000

Richland, Washington
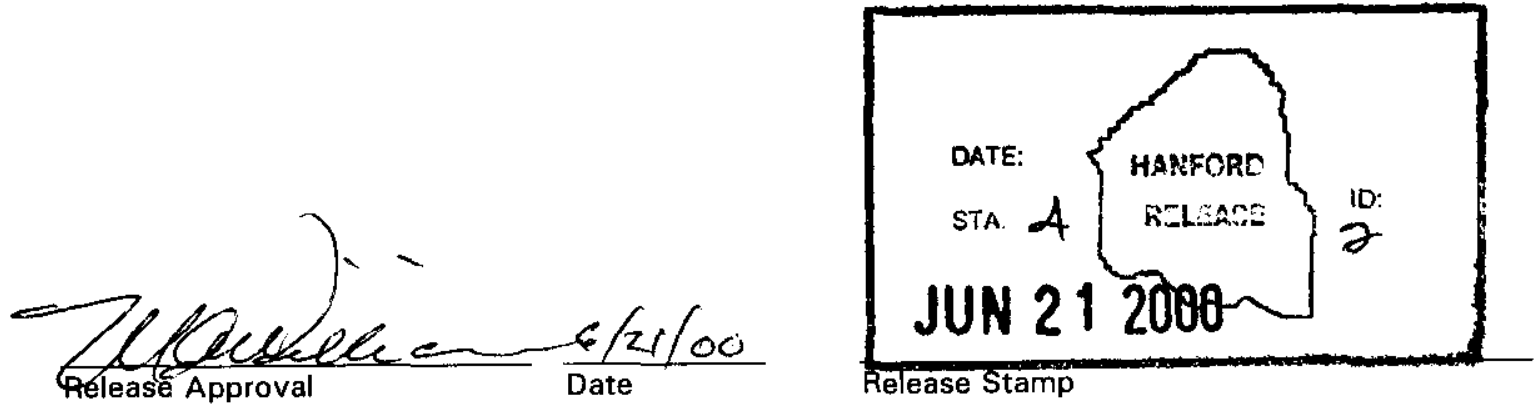
TRADEMARK DISCLAIMER

Reference herein to any specific commercial product, process,

or service by trade name, trademark, manufacturer, or

otherwise, does not necessarily constitute or imply its

endorsement, recommendation, or favoring by the United

States Government or any agency thereof or its contractors or subcontractors.

This report has been reproduced from the best available copy.

Printed in the United States of America

Total Pages: $\quad 15$

SNF- 3927, Nev3 


\section{RECORD OF REVISION}

(1) Document Number

$\mathrm{SNF}-3927$

Page 1

(2) Title

ASHCROET PRESSURE SWITCH - MONITOR FOR FLOW SCHE SUPPLY BOTTLE PRESSURE

Change Control Record

$\begin{array}{lll}\text { (3) Revision } & \text { (4) Description of Change - Replace, Add, and Delete Pages }\end{array}$

(7)

$0 \quad$ EDT 626276, INITIALLY RELEASED

1 ECN 653776, REVISION TO MEET SEL REV. 6

ECN 647508, REVISION TO MEET SEI REV. 6 a

3

RS ECN 661400, REVISION TO MEET PREVIOUS ECN TO SPEC

\begin{tabular}{|l|l|}
\hline \multicolumn{2}{|c|}{ Authorized for Release } \\
\hline (5) Cog. Engr. & (6) Cog. Mgr. Date \\
\hline C. Miska & T. Choho \\
\hline C. Miska & T. Choho \\
\hline C. Miska & T. Choho \\
\hline CD Nh Co/ $/ 9 / \infty$
\end{tabular}


Commercial Grade Item Upgrade Dedication Form

ECN No. NA CGI No.CGI-SNF-D-13-P5-030

Title: ASHCROFT PRESSURE SWITCH - MONITOR FOR LOW SCHe

SUPPLY BOTTLE PRESSURE

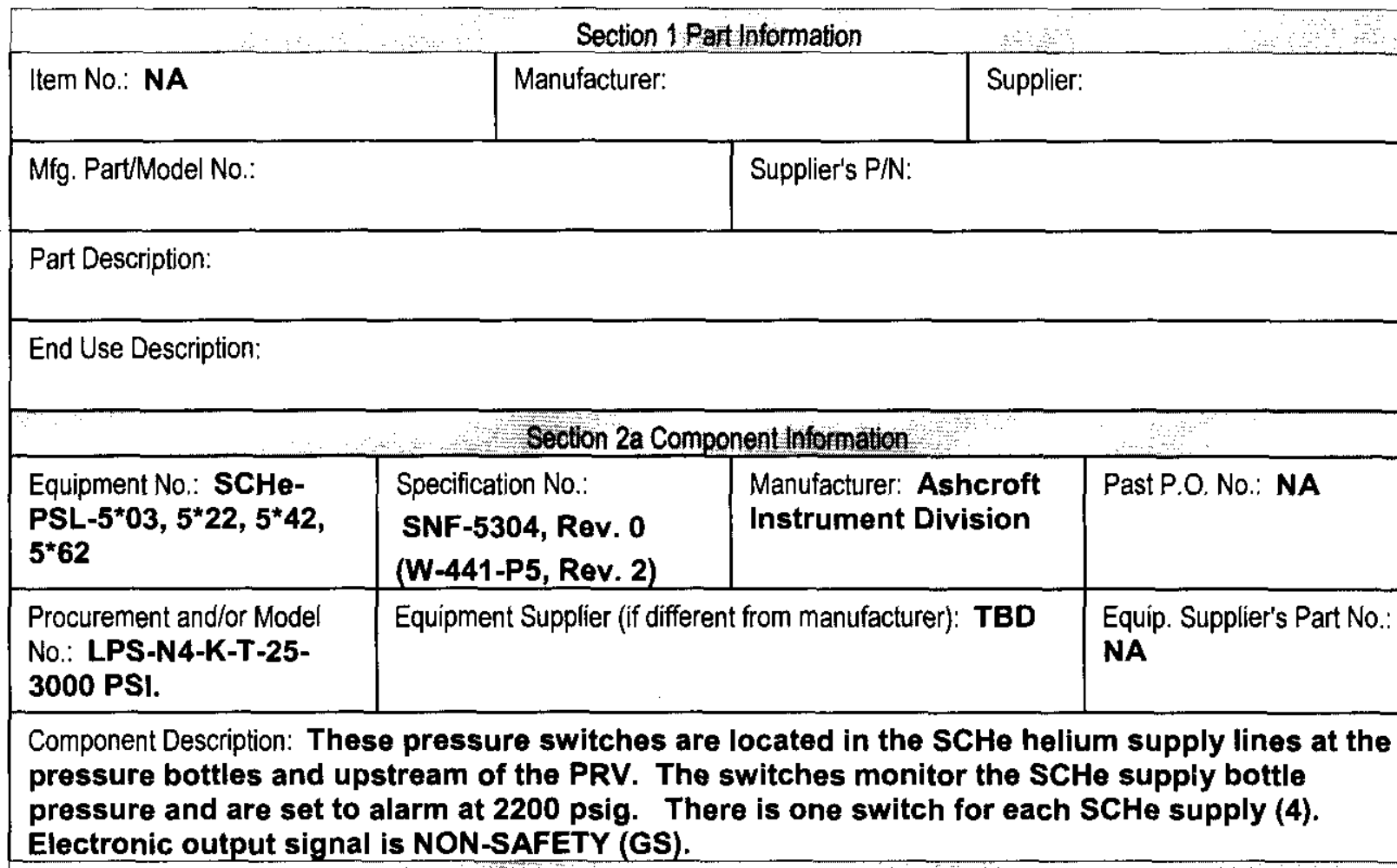

Section $2 \mathrm{~b}$ Qualified Vendor Suppller Survey

1. Is the Item available from a catalogue of a qualified NQA1 supplier? (coordinate with project CGI interface Engineer or BTR)

[ ] YES (go to \#2 below)

[X] NO (go to procedure step 5.3.2, proceed to dedicate ltem.)

If not available from a qualified NQA1 supplier, is it available from an ISO 9000 supplier? (coordinate with project CGI interface Engineer or BTR)

[ ] YES (go to \#2 below, then go to procedure step 5.3.2, proceed to dedicate item)

[X] NO (go to procedure step 5.3.2, proceed to dedicate Item.)

2. List of Candidate qualified suppliers or ISO 9000 suppliers

company name and type contact name phone

NA

3. Recommended Procurement Strategy (coordinate with project CGI interface Engineer or BTR):

NA

Secton 2 c C G Detemination

1. Question \#1: Is the Item subject to design or specification requirements that are unique to nuclear facilities or activities?

[ ] YES (the ltem is not commercial grade)

[X] NO (continue)

2. Question \#2: Is the Item used in applications other than nuclear facilities or activities?

[ ] NO (the item is not commercial grade)

[X] YES (continue) 


\section{SUPPLY BOTTLE PRESSURE}

3. Question \#3: Is the Item ordered from manufacturer/supplier on the basis or specifications set forth in the Published product information (e.g., manufacturer's catalog)?

[ ] $\mathrm{NO}$ (the Item is not commercial grade)

[X] YES (continue)

[X] All three criteria have been satisfied. The Item meets the definition of commercial grade.

\section{Section $2 \mathrm{~d}$ Reason for Dedlcation}

The above describediltem is being Dedicated for usie in the application cited for the following reason(s):

[X] Item is being purchased from a non ESL manufacturer supplier as commercial grade to be used in a Safety Class application.

[ ] Item is being purchased from a non ESL manufacturer supplier as commercial grade to be used in a Safety Significant application.

[ ] ltem was purchased from a non ESL manufacturer supplier as commercial grade to be used in a Safety Class application.

[ ] Item was purchased from a non ESL manufacturer supplier as commercial grade to be used in a Safety Significant application.

[ 1 Other ('like-for-like', similar, substitution, replacement evaluation)

\section{Section 3 Failure Effects Evaluation}

A. Part/Component Safety Function:

1. SCHe Pressure Boundary Integrity- Prevents helium leakage from the SCHe System.

2. Maintain pressure boundary before and after Seismic event.

B. Part/Component Functional Mode

Safety Function \#1:

[ ] Active - Mechanical or Electrical change of state is required to occur for the component to perform its safety function

[X] Passive - Change of state is not required for the component to perform its safety function

Safety Function \#2:

[ ] Active - Mechanical or Electrical change of state is required to occur for the component to perform its safety function.

[X] Passive - Change of state is not required for the component to perform its safety function Safety Function \#3:

[ ] Active - Mechanical or Electrical change of state is required to occur for the component to perform its safety function.

[ ] Passive - Change of state is not required for the component to perform its safety function

C. Host Component Safety Function (if applicable): NA

1. 
Title: ASHCROFT PRESSURE SWITCH - MONITOR FOR LOW SCHe SUPPLY BOTTLE PRESSURE

D. Failure Mechanisms(s) and the effects on component or system safety function (see worksheet 1):

1. Fracture of the switch body or of the piping connection resulting in loss of helium from the SCHe supply.

Section 4 Environmental \& Natural Phenomena Hazard Design

Environmental Qualification Required:

If yes: Environmental Qualification Requirements

Yes [ ]

Limiting Environmental Conditions:

No $[\mathbf{X}$ ]

Required Safety Functions:

Environmental Condition B

Qualification Period:

Natural Phenomena Hazard (NPH) Design Required:

Yes [X]

If yes: NPH Design Requirements

No [ ]

Performance Category: PC-3

NPH Design Req'ts.: Seismic Condition B

HNF-PRO-97, Rev. 0

Required Safety Functions: Pressure Boundary

W-441-P5, Rev. 2 Integrity

Section 5 Component Functional Classification

[X] Safety Class (SC) [ ] General Service

[ ] Safety Significant (SS)

If part/component classification is different from host component/system, document basis.

Pressure switch is pressure boundary Safety Class (SC).

Switch electronic output signal is General Service (GS).

Section 6 [reserved]

Section 7 [reserved]

Section 8 References (for Functional Classification)

National Codes/Standards: IEEE-344,

ISA-S5.1, -S5.4, -S18.1, -S20

Safety Analysis Report (SAR):

HNF-3553, Rev. Oa, Annex B

Drawings: H-1-82165, Rev. 2

HNF-SD-SNF-SEL-002, Rev.

6a CVDF-SSD-003

Vendor Manuals/Manufacturer/Supplier Information: Ashcroft Instrument Division, L Series Switches, Bulletin Se-12

Other: 


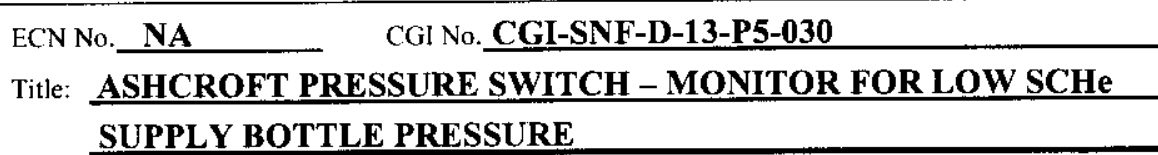

\begin{tabular}{|c|c|c|c|c|}
\hline 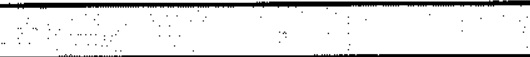 & \multicolumn{4}{|l|}{ Section 9 Grilical Characteristics } \\
\hline $\begin{array}{l}\text { Critical Characteristics } \\
\text { Verification Document:: Vendor } \\
\text { Specifications, } \\
\text { HNF-SD-SNF-SEL-002, Rev. 6a }\end{array}$ & Acceptance Criteria/Tolerances & $\begin{array}{c}\text { Acceptance } \\
\text { Method }\end{array}$ & ID & Function \\
\hline \multicolumn{5}{|c|}{ 1. Item Identification Critical Characteristics (necessary for reasonable assurance that the Item delivered is the Item specified) } \\
\hline $\begin{array}{l}\text { Component Number- } \\
\text { Procurement and/or Model } \\
\text { Number }\end{array}$ & $\begin{array}{l}\text { LPS-N4-K-T-25-3000 PSI, (Per } \\
\text { Procurement Package W-441- } \\
\text { P5, Rev. 2, Section G, Design } \\
\text { Data Sheet) } \\
\end{array}$ & $1, \mathrm{IN}$ & $\mathbf{x}$ & \\
\hline Nameplate - Manufacturer & Ashcroft Instruments & $1, \mathbf{I N}$ & $\mathbf{x}$ & \\
\hline Process Connection & $1 / 4$ Inch FNPT & $1, \mathbb{N}$ & $\mathbf{x}$ & \\
\hline Pressure Range & $0-3000$ psig & $1, \mathrm{IN}$ & $\mathbf{x}$ & \\
\hline Enclosure & NEMA 4 & $1, \mathbf{I N}$ & $\mathbf{x}$ & \\
\hline \multicolumn{5}{|c|}{ 2. Physical Critical Characteristics (necessary for reasonable assurance that the Item delivered is the Item specified) } \\
\hline Material, Body & Stainless Steel (Note 4) & 1, IN 1,T & $\mathbf{x}$ & \\
\hline Material, Process Connection & Stainless Steel (Note 4) & 1, IN 1,T & $\mathbf{x}$ & \\
\hline \multicolumn{5}{|c|}{$\begin{array}{l}\text { 3. Performance Critical Characteristics (necessary \& sufficient for reasonable assurance that the Item will perform its intended } \\
\text { safety function(s)) }\end{array}$} \\
\hline Pressure Boundary Integrity & \begin{tabular}{|l|} 
No Leakage at Test Pressure \\
of 4400 psig (No Bubbles) Note \\
3 \\
\end{tabular} & $1, \mathrm{~T}$ & & $\mathbf{x}$ \\
\hline Setpoint/Repeatability & $\begin{array}{l}\text { Nominal } 2200 \text { psig } /+1-1 \% \text { of } \\
\text { Range }\end{array}$ & $1, T$ & & $\mathbf{x}$ \\
\hline Environmental & Note 1 & & & \\
\hline Seismic Condition B & Note 2 & $1, T$ & & $x$ \\
\hline \multicolumn{2}{|c|}{$\begin{array}{l}\text { 2. Maintain pressure boundary before and after Seismic event. } \\
\text { W-441-P5, Rev. 2, Appendix I, page I-2, provides a seismic } \\
\text { testing plan for these components at a seismic spectra TBD. } \\
\text { Equipment that has been shaker-table tested should not be } \\
\text { installed in a plant (Ref. IEEE Standard } 344-1984 \text {, Section } 7 \text { ). } \\
\text { Consequently, the seismic test constitutes a destructive test. }\end{array}$} & \multicolumn{3}{|c|}{$\begin{array}{l}\text { Acceptance Method: } \\
\text { 1. Special Test and Inspection } \\
\text { 1,IN for Inspection } \\
1, T \text { for Test } \\
\text { 2. Commercial Grade Survey } \\
\text { 3. Source Verification } \\
\text { 4. Vendor/ltem History }\end{array}$} \\
\hline \multicolumn{5}{|c|}{$\begin{array}{l}\text { 3. Pressure test at } 110 \% \text { of design pressure. } \\
\text { 4. Material verification acceptance method may be by either } \\
\text { inspection or test. }\end{array}$} \\
\hline
\end{tabular}


Approvals:
Designated Engineeg
Design Authority:

Design Authority: $10 / 6 / 0^{\circ}$

QA Engineer:_L $6(20)$ 


\begin{tabular}{|c|c|}
\hline Commercial Grade Item Upgrade Dedication Form & SNF-3927, Rev. 3 \\
\hline ECN No. NA $\quad$ CGI No.CGI-SNF-D-13-P5-030 & \multirow[t]{3}{*}{ Page 6 of 12} \\
\hline Title: ASHCROFT PRESSURE SWITCH - MONITOR FOR LOW SCHe & \\
\hline SUPPLY BOTTLE PRESSURE & \\
\hline
\end{tabular}

\begin{tabular}{|c|c|c|}
\hline \multirow{2}{*}{-7} & \multirow{2}{*}{$\begin{array}{c}\text { WORKSHEET } 1 \\
\text { DETERMINATION OF FAILURE MECHANISMSIMODES } \\
\text { sECTION } 1\end{array}$} & 1ODES \\
\hline & & SECTION 1 \\
\hline \multicolumn{3}{|r|}{$\begin{array}{l}\text { Applicable to Component } \\
\text { under Evaluation }\end{array}$} \\
\hline Fracture & $\begin{array}{l}\text { Separation of a solid accompanied by little or no } \\
\text { macroscopic plastic deformation. }\end{array}$ & $\begin{array}{l}\text { Yes [X] No [ ]; If Yes, indicate } \\
\text { failure Mode. Failure of Transmitter } \\
\text { Body or the Process Connection }\end{array}$ \\
\hline Corrosion & \multirow{2}{*}{$\begin{array}{l}\text { The gradual deterioration of a material due to } \\
\text { chemical or electrochemical reactions, such as } \\
\text { oxidation, between the material and its environment. } \\
\text { Destruction of materials by the abrasive action of } \\
\text { moving fluids, usually accelerated by the presence } \\
\text { of solid particles carried with the fluid. }\end{array}$} & $\begin{array}{l}\text { Yes [ ] No [X]; If Yes, indicate } \\
\text { failure Mode. }\end{array}$ \\
\hline Erosion & & $\begin{array}{l}\text { Yes [] No [X]; If Yes, indicate } \\
\text { failure Mode. }\end{array}$ \\
\hline Open Circuit & $\begin{array}{l}\text { An electrical circuit that is unintentionally broken so } \\
\text { that there is no complete path for current flow. }\end{array}$ & $\begin{array}{l}\text { Yes [ ] No [X]; If Yes, indicate } \\
\text { failure Mode. }\end{array}$ \\
\hline Short Circuit & \multirow{3}{*}{$\begin{array}{l}\text { An abnormal connection by which an electrical } \\
\text { current is connected to ground, or to some } \\
\text { conducting body, resulting in excessive current flow. } \\
\text { Clogging of a filtering medium resulting in the } \\
\text { inability to perform its purification function or } \\
\text { blockage of flow. } \\
\text { Binding of a normally moving item through excessive } \\
\text { pressure, temperature, friction, jamming. }\end{array}$} & $\begin{array}{l}\text { Yes [ ] No [X]; If Yes, indicate } \\
\text { failure Mode. }\end{array}$ \\
\hline Blockage & & $\begin{array}{l}\text { Yes [ ] No [X]; If Yes, indicate } \\
\text { failure Mode. }\end{array}$ \\
\hline Seizure & & $\begin{array}{l}\text { Yes [ ] No [X]; If Yes, indicate } \\
\text { failure Mode. }\end{array}$ \\
\hline Unacceptable Vibration & $\begin{array}{l}\text { Mechanical oscillations produced are beyond the } \\
\text { defined permissible limits due to unbalancing, poor } \\
\text { support, or rotation at critical speeds. }\end{array}$ & $\begin{array}{l}\text { Yes [ ] No [X]; If Yes, indicate } \\
\text { failure Mode. }\end{array}$ \\
\hline Loss of Properties & $\begin{array}{l}\text { A loss of mechanical and physical properties of a } \\
\text { material due to exposure to high temperatures, } \\
\text { radiation exposure. }\end{array}$ & $\begin{array}{l}\text { Yes [ ] No [X]; If Yes, indicate } \\
\text { failure Mode. }\end{array}$ \\
\hline Excess Strain & $\begin{array}{l}\text { Under the action of excessive external forces the } \\
\text { material of the part has been deformed or distorted. }\end{array}$ & $\begin{array}{l}\text { Yes [ ] No [X]; If Yes, indicate } \\
\text { failure Mode. }\end{array}$ \\
\hline Mechanical Creep & $\begin{array}{l}\text { From prolonged exposure to high temperature and } \\
\text { stress, the object will show a slow change in its } \\
\text { physical (shape and dimension) and mechanical } \\
\text { characteristics. }\end{array}$ & $\begin{array}{l}\text { Yes [ ] No [X]; If Yes, indicate } \\
\text { failure Mode. }\end{array}$ \\
\hline Ductile Fracture & $\begin{array}{l}\text { characteristics. } \\
\text { Fracture characterized by tearing of metal } \\
\text { accompanied by appreciable gross plastic } \\
\text { deformation. }\end{array}$ & $\begin{array}{l}\text { Yes [ ] No [X]; If Yes, indicate } \\
\text { failure Mode. }\end{array}$ \\
\hline \multicolumn{3}{|c|}{$\begin{array}{l}\text { SECTION } 2 \text { Addlitonal Fallure Wodes Applicable to the Component Under Evaluation } \\
\text { inection/Body Break }\end{array}$} \\
\hline
\end{tabular}


CHECKLIST 1

ACCEPTANCE METHOD 1

SPECIAL TEST/INSPECTION VERIFICATION

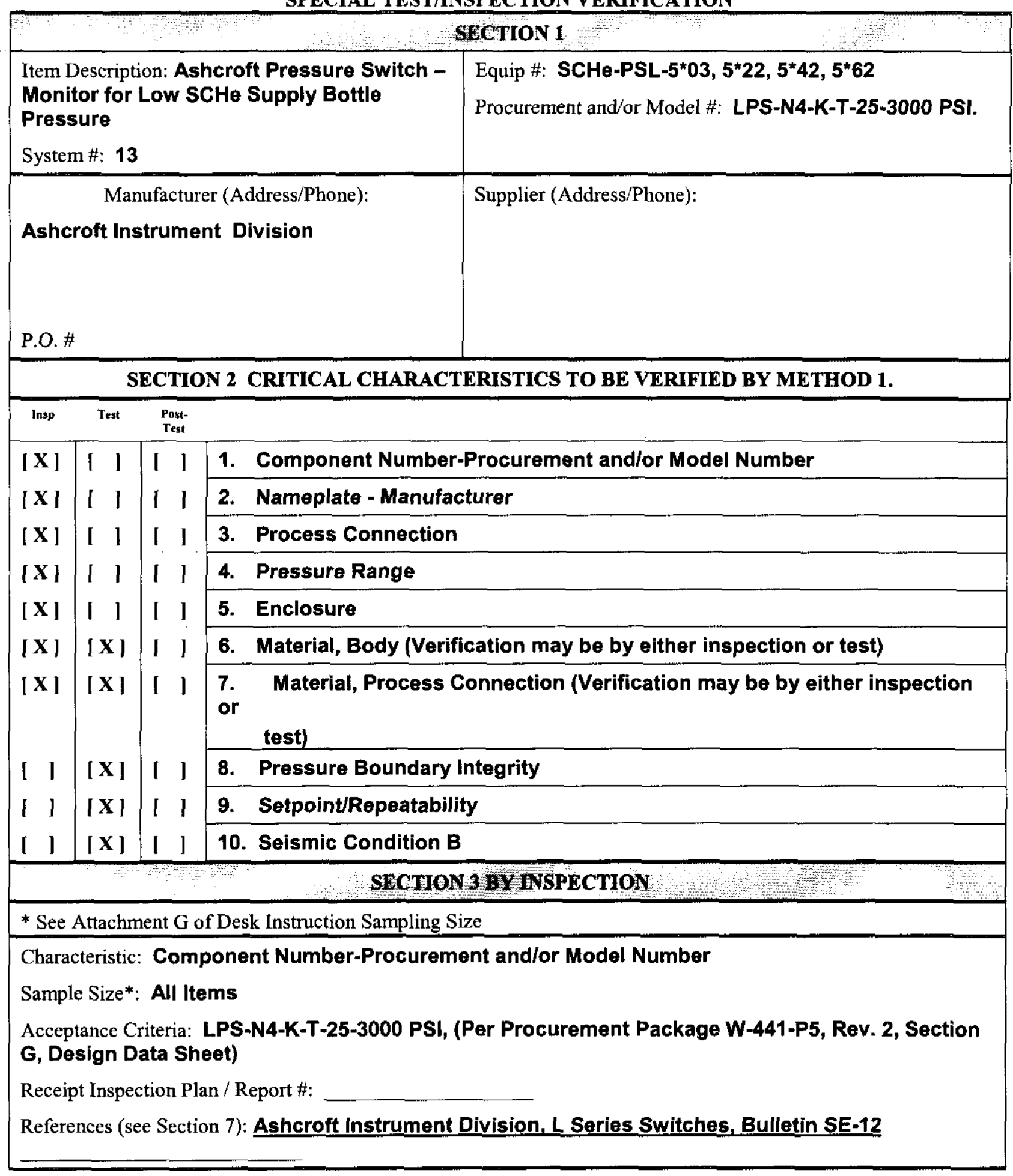




\begin{tabular}{ll} 
ECN No. NA CGI No.CGI-SNF-D-13-P5-030 \\
Title: $\frac{\text { ASHCROFT PRESSURE SWITCH -MONITOR FOR LOW SCHe }}{\text { SUPPLY BOTTLE PRESSURE }}$ \\
\hline
\end{tabular}

Characteristic: Nameplate - Manufacturer

Sample Size*: All Items

Acceptance Criteria: Ashcroft Instruments

Receipt Inspection Plan/ Report \#:

References (see Section 7):

Characteristic: Process Connection

Sample Size*: All Items

Acceptance Criteria: $1 / 4$ Inch FNPT

Receipt Inspection Plan / Report \#:

References (see Section 7):

Characteristic: Pressure Range

Sample Size*: All Items

Acceptance Criteria: 0-3000 psig

Receipt Inspection Plan / Report \#:

References (see Section 7):

Characteristic: Enclosure

Sample Size*: All Items

Acceptance Criteria: NEMA 4

Receipt Inspection Plan / Report \#:

References (see Section 7):

Characteristic: Material, Body

Sample Size*: Normal Sampling Size

Acceptance Criteria: Stainless Steel

Receipt Inspection Plan / Report \#:

References (see Section 7):

Characteristic: Material, Process Connection

Sample Size*: Normal Sampling Size

Acceptance Criteria: Stainless Steel

Receipt Inspection Plan / Report \#:

References (see Section 7): 


\begin{tabular}{|l|l|}
\hline \multicolumn{2}{|c|}{ SECTION 4BY SPECIAL TEST } \\
\hline * See Attachment G of Desk Instruction Sampling Size \\
\hline Test To Be Performed by: & Number of Items to be Tested: \\
{$[\quad$ ] Purchaser } & Test/Inspection Location: \\
{$[\quad$ ] Supplier/Manufacturer** } \\
[ ] Other
\end{tabular}

**If Supplier/Manufacturer or Other, Refer to CGI Checklist-2 for Support Information 


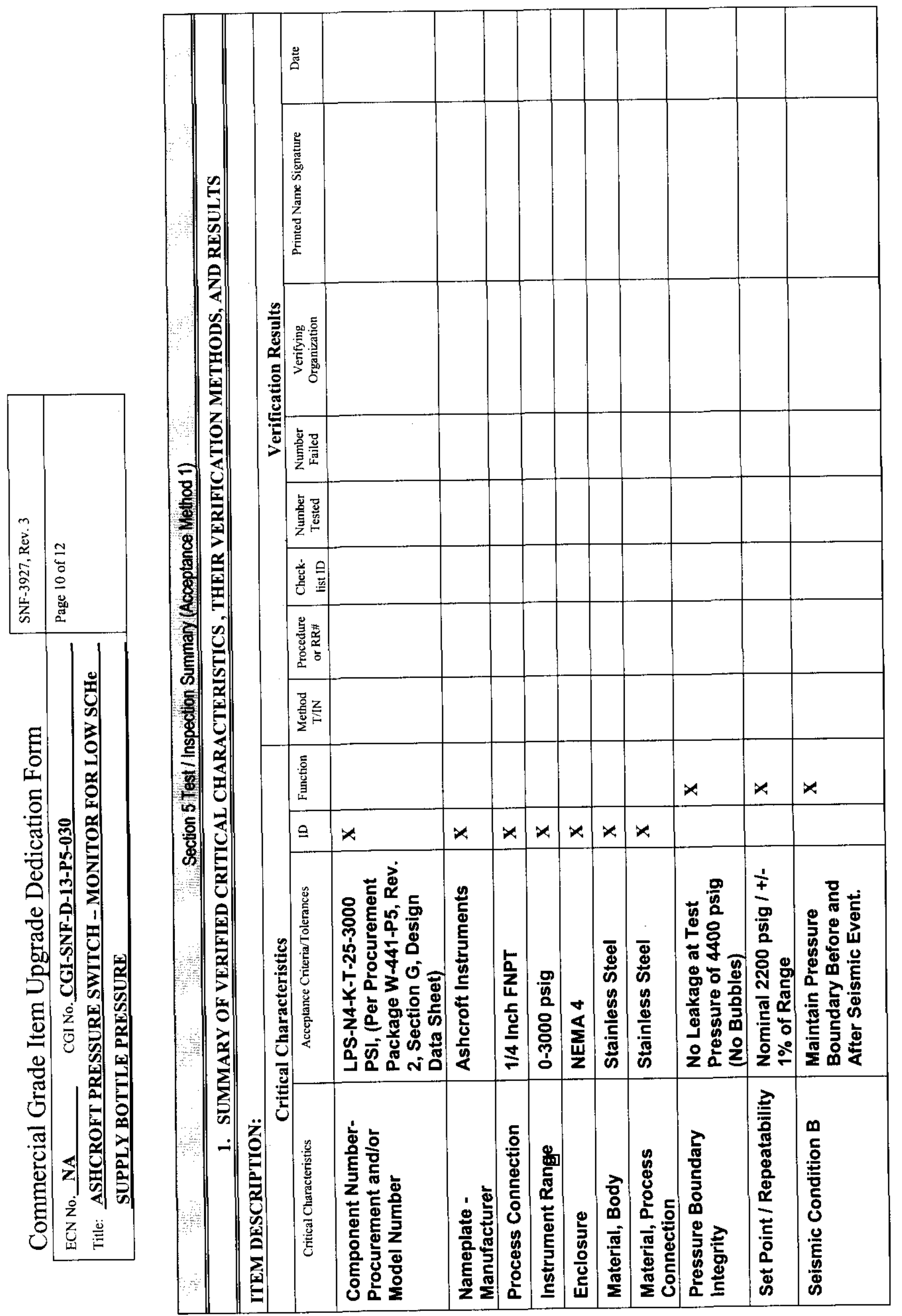




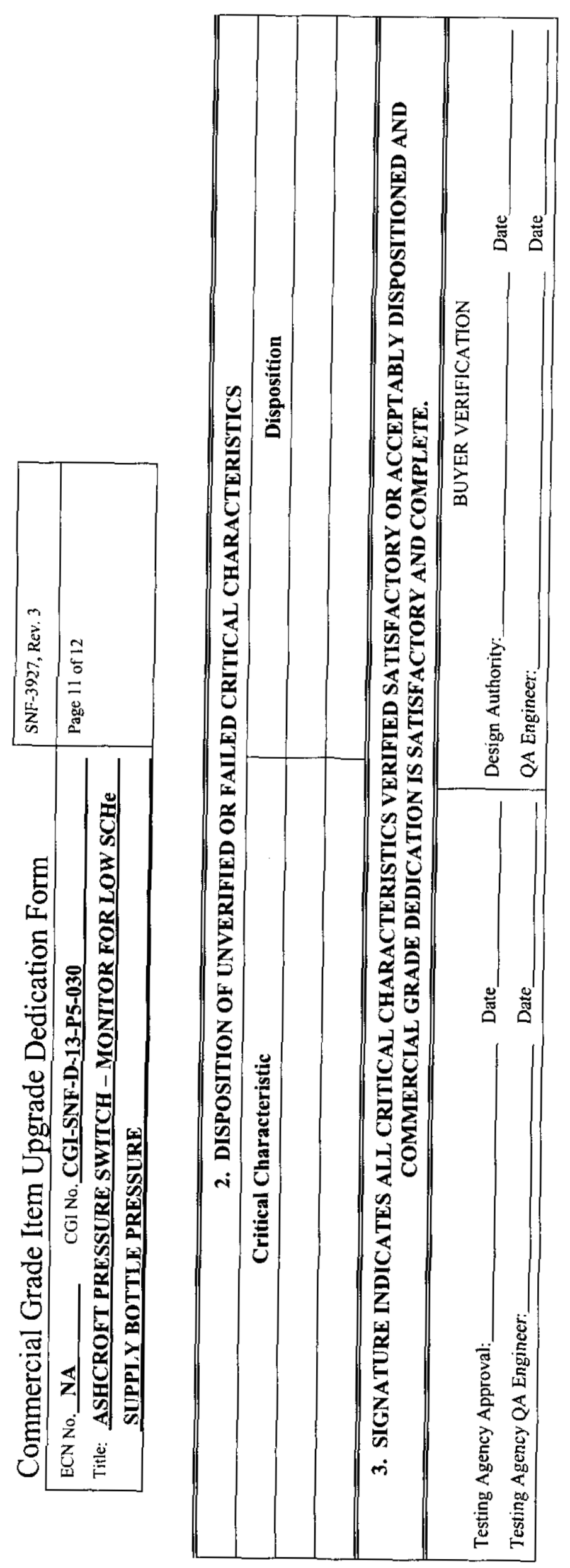

$\frac{8}{8}$

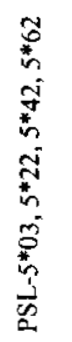


Commercial Grade Item Upgrade Dedication Form

ECN No. NA CGI No.CGI-SNF-D-13-P5-030

Title: ASHCROFT PRESSURE SWITCH - MONITOR FOR LOW SCHe

\section{SUPPLY BOTTLE PRESSURE}

SNF-3927, Rev. 3

Page 12 of 12

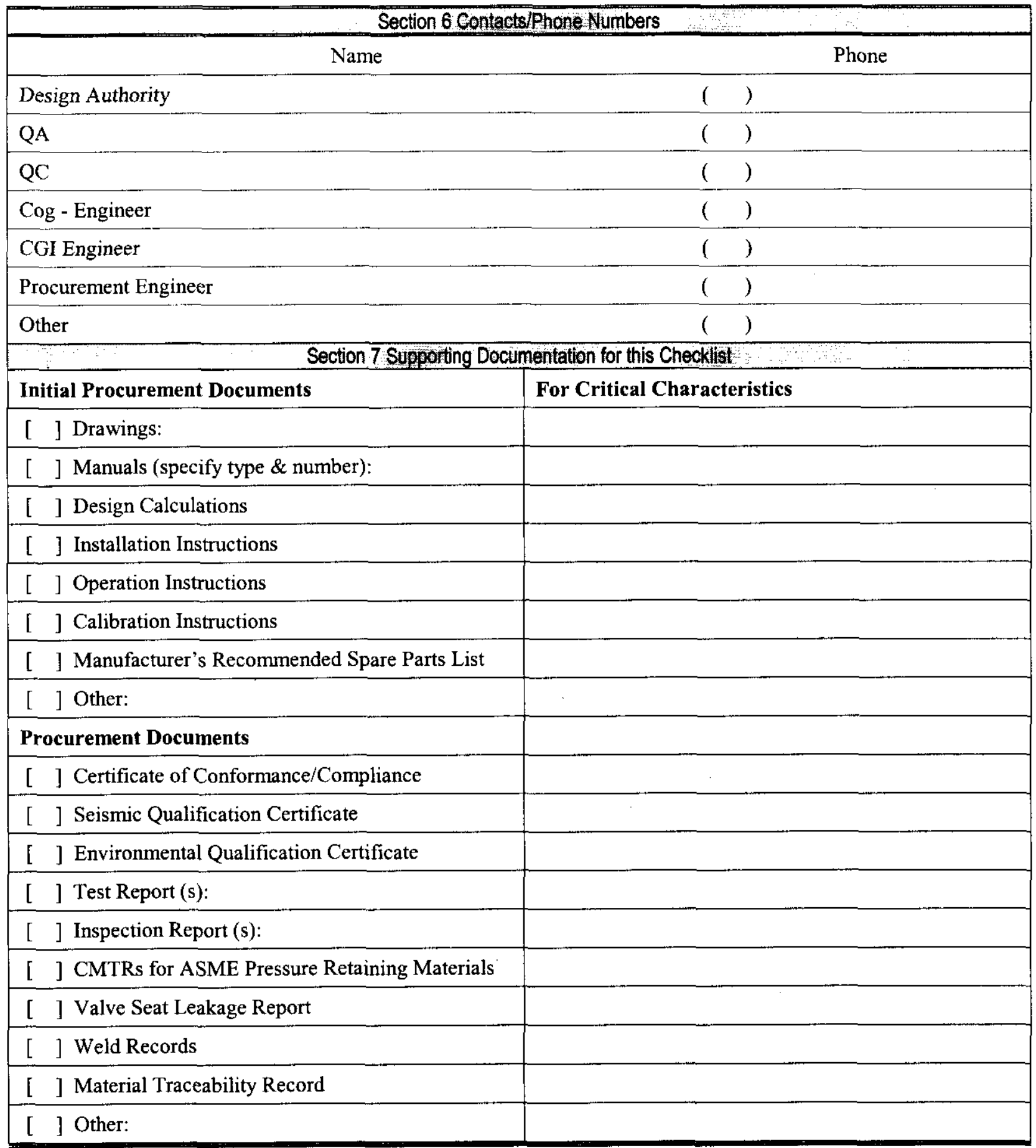

